This is an electronic reprint of the original article. This reprint may differ from the original in pagination and typographic detail.

Author(s): Airaksinen, Tuomas; Heikkola, Erkki

Title: Multiobjective muffler shape optimization with hybrid acoustics modelling

Year: $\quad 2011$

Version:

Please cite the original version:

Airaksinen, T., \& Heikkola, E. (2011). Multiobjective muffler shape optimization with hybrid acoustics modelling. Journal Of Acoustical Society of America, 130(3), 13591369. https://doi.org/10.1121/1.3621119

All material supplied via JYX is protected by copyright and other intellectual property rights, and duplication or sale of all or part of any of the repository collections is not permitted, except that material may be duplicated by you for your research use or educational purposes in electronic or print form. You must obtain permission for any other use. Electronic or print copies may not be offered, whether for sale or otherwise to anyone who is not an authorised user. 


\title{
Multiobjective muffler shape optimization with hybrid acoustics modeling
}

\author{
Tuomas Airaksinen ${ }^{\text {a) }}$ \\ Department of Mathematical Information Technology, University of Jyväskylä, P.O. Box 35, \\ FIN-40014 Jyväskylä, Finland \\ Erkki Heikkola \\ Numerola Ltd., P.O. Box 126, FIN-40101 Jyväskylä, Finland
}

(Received 14 May 2010; revised 23 June 2011; accepted 5 July 2011)

\begin{abstract}
This paper considers the combined use of a hybrid numerical method for the modeling of acoustic mufflers and a genetic algorithm for multiobjective optimization. The hybrid numerical method provides accurate modeling of sound propagation in uniform waveguides with non-uniform obstructions. It is based on coupling a wave based modal solution in the uniform sections of the waveguide to a finite element solution in the non-uniform component. Finite element method provides flexible modeling of complicated geometries, varying material parameters, and boundary conditions, while the wave based solution leads to accurate treatment of non-reflecting boundaries and straightforward computation of the transmission loss (TL) of the muffler. The goal of optimization is to maximize TL at multiple frequency ranges simultaneously by adjusting chosen shape parameters of the muffler. This task is formulated as a multiobjective optimization problem with the objectives depending on the solution of the simulation model. NSGA-II genetic algorithm is used for solving the multiobjective optimization problem. Genetic algorithms can be easily combined with different simulation methods, and they are not sensitive to the smoothness properties of the objective functions. Numerical experiments demonstrate the accuracy and feasibility of the model-based optimization method in muffler design. (C) 2011 Acoustical Society of America. [DOI: 10.1121/1.3621119]
\end{abstract}

PACS number(s): 43.50.Gf, 43.20.Mv, 43.20.Hq, 43.55.Ka [AH] Pages: 1359-1369

\section{INTRODUCTION}

In ductwork, the purpose of a muffler or a silencer is to attenuate certain sound frequencies and thus prevent the propagation of noise to the duct openings (see Bodén and Glav ${ }^{1}$ ). They are typically divided into two categories depending on the physical principle of sound attenuation. Reactive mufflers are based on the reflection of sound from suitable geometrical shapes and resonators. Dissipative mufflers, on the other hand, attenuate sound by absorbing materials such as wool and foams. Both types have specific benefits and drawbacks, and it depends on the application which type of attenuation is best suited for the problem at hand. Often mufflers are designed to have both reactive and dissipative properties. Here, we concentrate on the modeling and shape optimization of reactive silencers, but the same approach is applicable also to the material or topology optimization of dissipative mufflers (e.g., see Lee and $\mathrm{Kim}^{2}$ ).

Different kinds of mufflers are necessary components in the attenuation of noise in automobiles, air-conditioning and ventilation systems as well as large-scale industrial engines, for example. They are also used to attenuate unwanted lowfrequency pulsations in many industrial applications. Especially large-scale industrial attenuators are expensive to build and study experimentally.

\footnotetext{
a) Author to whom correspondence should be addressed. Electronic mail: tuomas.a.airaksinen@jyu.fi
}

Development of numerical methods for duct acoustics and mufflers has been active since the 1970s. Most simulation studies and software in duct acoustics are using the transfer matrix method (TMM) or the four-pole method, which is based on the assumption of plane wave propagation (see, e.g., Munjal ${ }^{3}$ and Bodén and Glav ${ }^{1}$ for more details). This assumption is reasonable in the low-frequency region, which is relevant in many engine applications. Finite element and boundary element methods have also been used for duct acoustics in many studies (see Bilawchuk and $\mathrm{Fyfe}^{4}$ and Mehdizadeh and Paraschivoiu ${ }^{5}$ ). Finite element methods (FEMs) provide more accurate results in a wider range of frequencies than one-dimensional plane wave methods. They are also more flexible with respect to geometrical details, varying material parameters, and boundary conditions. Bilawchuk and $\mathrm{Fyfe}^{4}$ report comparisons on different methods for muffler modeling and conclude that FEM is best suited for this application.

In this work, we use a hybrid numerical method introduced by Kirby ${ }^{6}$ to model sound propagation in ducts. This method is based on combining wave based modal solution in uniform parts of the ductwork to a finite element solution in non-uniform parts, such as mufflers. Mode matching is used to couple the two solution representations along given boundaries. Hybrid numerical method for duct acoustics is closely related to the use of Dirichlet-to-Neumann boundary condition in finite element modeling $\left(\right.$ Astley $\left.^{7}\right)$. It provides accurate anechoic boundary condition on the outgoing boundary of the non-uniform part also in the high-frequency cases where the 
assumption of plane wave propagation is no longer valid. Furthermore, it leads to a straightforward computation of the transmission loss (TL) of non-uniform components which is very useful for the evaluation of various muffler designs.

FEM is more time-consuming and technically difficult than TMM, which makes it less attractive in practical muffler engineering (Gerges et al. ${ }^{8}$ ). One-dimensional models remain useful in fast evaluation of muffler prototypes, but we believe that model-based optimization should be based on more accurate and reliable modeling methods, such as FEM. Otherwise the optimization results may be misleading. One of the main aims of this work is to develop the practical combination of finite element based modeling and optimization algorithms as well as to demonstrate the feasibility of the approach.

A natural motive for computer simulations is to be able to study and optimize devices without time-consuming and expensive experiments. Also computer simulations may be inefficient and lead to inadequate results if they are based only on trial and error methodology. If there are several (five or more) design parameters which affect the final outcome of a simulation model, it is often very difficult, even for an expert, to find the optimal set of parameters just by intuition. There is an obvious need for an automated optimization procedure in addition to the simulation model.

To our knowledge the model-based shape optimization of acoustic mufflers has first been studied by Bernhard, ${ }^{9}$ who used both the transfer matrix method and the finite element method for acoustic modeling. More recently, the finite element based shape optimization has been considered by Barbieri and Barbieri ${ }^{10}$ and Kuskinen et al., ${ }^{11}$ and we apply here a closely related approach. The model-based shape optimization of different types of mufflers with the transfer matrix method and various optimization algorithms has been considered in a series of articles by Chiu et al. ${ }^{12-16}$

The main stages of our model-based optimization are considered in Sec. IV, and they basically coincide with the ones listed in Barbieri and Barbieri. ${ }^{10}$ There are, however, significant differences in the realization of the simulation and optimization methods. Instead of using just the finite element method, we complement it with the hybrid method, which provides more accurate treatment of the non-reflecting boundaries and straightforward computation of the transmission loss of the muffler. In Barbieri and Barbieri, ${ }^{10}$ the objective is also to maximize TL at multiple frequency ranges simultaneously, but this is formulated as a single objective optimization problem. Thus, their result is only one representative from the set of Pareto-optimal solutions. We solve the actual multiobjective optimization problem, which gives the whole family of Pareto-optimal solutions with contradicting objectives (separate frequency ranges). Another notable difference is that in Barbieri and Barbieri ${ }^{10}$ a gradient-based optimization method is used while we use genetic algorithm which does not require gradient information. Gradient-based methods are usually computationally more efficient, but they require a method to compute derivatives of the objective function with respect to control variables. They are also more sensitive to irregularities in the objective function (non-smoothness, discontinuities). Therefore, we prefer here to apply more robust gradient-free methods even though they probably lead to higher computational cost.

We study the performance of the simulation and optimization method with four test cases, which are taken from Gerges et al., ${ }^{8}$ Chiu et al., ${ }^{12}$ and Chiu. ${ }^{17}$ The goals of the tests are first to validate the simulation model with practical measurements and to study the accuracy of the outgoing boundary condition. We use the first test case from Gerges et $a l .{ }^{8}$ for this purpose. The results also demonstrate the better accuracy of the FEM method in comparison with the TMM. Three optimization test cases are used to find optimal dimensions or perforation parameters for typical reflective mufflers. The first optimization case is taken from Gerges et al., ${ }^{8}$ and it represents a muffler with a perforated duct, where shape and perforation parameters are optimized. The second optimization test case is taken from Chiu, ${ }^{17}$ and it represents a more complicated reverse-flow type muffler geometry. The third optimization case is taken from Chiu et al., ${ }^{12}$ and it is used to demonstrate the ability of optimizing the muffler in the case with several modes propagating in the ductwork (high-frequency case).

The rest of the article is organized as follows: Sec. II gives the mathematical formulation of the acoustic wave propagation and the wave based representation of the solution in the uniform parts of the ductwork. The hybrid numerical method with the mode matching between finite element approximation and wave based modal solutions is described in Sec. III. The model-based optimization approach as well as its practical realization with the genetic optimization algorithm are considered in Sec. IV. Numerical tests and results are reported and analyzed in Sec. V, and main conclusions based on the results are collected in Sec. VI.

\section{MATHEMATICAL FORMULATION}

We consider the propagation of sound waves in a homogeneous and inviscid fluid with negligible heat transfer (adiabatic). By further assuming that the pressure and density variations are small perturbations of the static state and the fluid is stagnant, we are able to linearize the governing equations to obtain the linear wave equation

$$
c^{2} \nabla \cdot \frac{1}{\rho} \nabla \tilde{p}-\frac{1}{\rho} \frac{\partial^{2} \tilde{p}}{\partial t^{2}}=0
$$

where $\tilde{p}$ is the acoustic pressure fluctuation and $c$ is the speed of sound (see, e.g., Rienstra and Hirschberg ${ }^{18}$ ). By assuming harmonic time-dependence $\tilde{p}(\mathbf{x}, t)=p(\mathbf{x}) e^{-i w t}$ with angular frequency $\omega$, Eq. (1) leads to the Helmholtz equation

$$
-\nabla \cdot \frac{1}{\rho} \nabla p-\frac{k^{2}}{\rho} p=0,
$$

where $k$ is the wave number.

Our goal is to simulate numerically the acoustic effect of a non-uniform perturbation in a uniform ductwork. This problem has recently been considered by Kirby, ${ }^{6,19}$ and we apply the hybrid numerical formulation and method introduced in his articles. Such a system is illustrated in Fig. 1, 


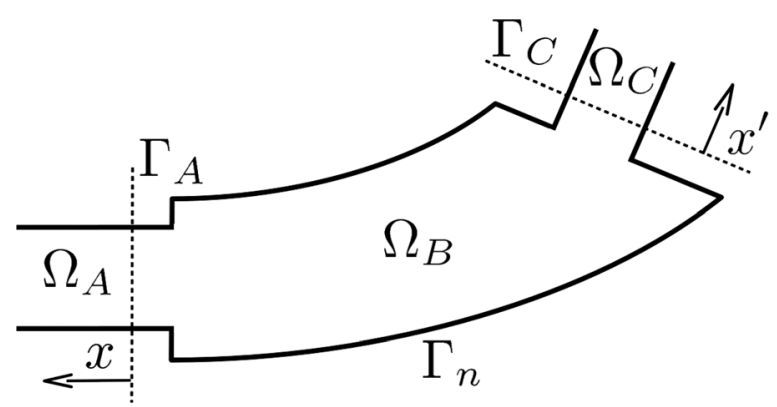

FIG. 1. The crosscut illustration of a duct system in a general case: inlet duct $\Omega_{A}$, muffler component of arbitrary shape $\Omega_{B}$ and outlet duct $\Omega_{C}$.

where the geometry is divided into three parts: The inlet and outlet ducts, denoted by $\Omega_{A}$ and $\Omega_{C}$, and the non-uniform part, denoted by $\Omega_{B}$. The inlet and outlet ducts are assumed to have uniform cross sections and they are separated from the non-uniform part by the planes $\Gamma_{A}$ and $\Gamma_{C}$ which are perpendicular to the axes of propagation of the ducts $\Omega_{A}$ and $\Omega_{C}$, respectively. These axes are denoted by $x$ and $x^{\prime}$ and are shown in Fig. 1, and we assume that $x=0$ at $\Gamma_{A}$ and $x^{\prime}=0$ at $\Gamma_{C}$. The number of inlet and outlet ducts can be larger than one, but for simplicity we restrict our attention to the case with one inlet and one outlet.

At the walls of the duct system we assume the soundhard boundary condition

$$
\mathbf{n} \cdot \nabla p=0 \quad \text { on } \Gamma_{n},
$$

but the hybrid numerical method used here would allow more general boundary conditions of the form $a \mathbf{n} \cdot \nabla p+b p=0$ with coefficients $a$ and $b$ independent of the axial coordinates of the ducts. In the non-uniform part of the system, also the boundary conditions can be non-uniform.

\section{A. Solutions in the uniform ducts}

Solution of the Helmholtz equation Eq. (2) in the uniform sections $\Omega_{A}$ and $\Omega_{C}$ can be represented by infinite sums of the form

$$
\begin{aligned}
& p_{A}(r, \theta, x)=\sum_{j=0}^{\infty} A_{j} \Phi_{j}(r, \theta) e^{-i \lambda_{j} x}+\sum_{j=0}^{\infty} F_{j} \Phi_{j}(r, \theta) e^{i \lambda_{j} x} \\
& p_{C}\left(r, \theta, x^{\prime}\right)=\sum_{j=0}^{\infty} B_{j} \Psi_{j}(r, \theta) e^{-i \gamma_{j} x^{\prime}}+\sum_{j=0}^{\infty} C_{j} \Psi_{j}(r, \theta) e^{i \gamma_{j} x^{\prime}}
\end{aligned}
$$

The functions $\Phi_{j}$ and $\Psi_{j}$ are the eigenfunctions of the Laplace operator corresponding to the uniform cross section $\Gamma_{A}$ and $\Gamma_{C}$ (see Rienstra and Hirschberg ${ }^{18}$ ). They are multiplied by exponential terms with axial wave numbers $\gamma_{j}$ and $\lambda_{j}$, and the modal amplitudes $F_{j}, A_{j}, B_{j}$, and $C_{j}$. Only a finite number of wave numbers $\gamma_{j}$ and $\lambda_{j}$ are real while the remaining values are purely imaginary. Let us denote these finite numbers by $n_{A}$ and $n_{C}$. The modes corresponding to the real-valued wave numbers are propagating and are called cut-on (see, e.g., Kirby ${ }^{6}$ and Rienstra and Hirschberg ${ }^{18}$ ). The modes corresponding to the imaginary wave numbers decay exponentially with respect to the axial coordinate and are called cut-off.

The sound transmission loss (TL) of the non-uniform part $\Omega_{B}$ is defined as the ratio of the transmitted and incident sound powers. It is obtained from the coefficients $F_{j}$ and $B_{j}$ by

$$
T L(f)=-10 \log _{10} \frac{\rho_{A} \sum_{m=0}^{n_{A}} \gamma_{m} H_{m}\left|B_{m}\right|^{2}}{\rho_{C} \sum_{m=0}^{n_{C}} \lambda_{m} I_{m}\left|F_{m}\right|^{2}},
$$

where $I_{n}=\int_{\Gamma_{A}}\left|\Phi_{n}\right|^{2} d S$ and $H_{m}=\int_{\Gamma_{B}}\left|\Psi_{m}\right|^{2} d S$ (Kirby ${ }^{6}$ ). Here, the coefficients $F_{j}$ determine the incoming sound from inlet duct. The equal modal energy density (EMED) assumption, i.e., $\left|F_{n}\right|^{2} I_{n}=\left|F_{m}\right|^{2} I_{m}$ for all $m, n$, has been chosen for the inlet as incident sound source, as it is a good representation of the sound field emanating from a fan in a ventilation system; see Kirby and Lawrie. ${ }^{20}$ For EMED, the incoming modal amplitudes can be calculated from the formula

$$
\left|F_{n}\right|^{2}=\frac{I_{0}}{I_{n} \sum_{m=0}^{n_{A}} \lambda_{m}}
$$

Modal amplitude coefficients $A_{j}$ correspond to the sound that is reflected back from the muffler, $B_{j}$ correspond to the sound propagating to the outlet duct, and $C_{j}$ correspond to the sound that is reflected back from outlet duct. By setting $C_{j}=0$ on $\Gamma_{C}$ for all $j$, a perfectly non-reflecting boundary is imposed on $\Gamma_{C}\left(\right.$ Astley $\left.^{7}\right)$. With the EMED assumption Eq. (6), we can write the transmission loss in a simpler form

$$
T L(f)=-10 \log _{10} \frac{\rho_{A} \sum_{m=0}^{n_{B}} \gamma_{m} H_{m}\left|B_{m}\right|^{2}}{\rho_{C} I_{0}} .
$$

The shape of the uniform cross sections of the ducts $\Omega_{A}$ and $\Omega_{C}$ is not restricted, but often the shape is either rectangular or circular. In these cases, it is possible to derive explicit representations for the eigenfunctions $\Phi_{j}$ and $\Psi_{j}$ in terms of trigonometric or Bessel functions (Rienstra and Hirschberg ${ }^{18}$ ). We concentrate here to cylindrical ducts where the modes are of the form

$$
\Phi_{j}(r, \theta)=N_{j} J_{m_{j}}\left(k_{r j} r\right) e^{i m_{j} \theta}
$$

where $J_{m_{j}}(x)$ is order $m_{j}$ Bessel function of the first kind and $k_{r j}$ is the radial wave number. The radial wave number $k_{r j}$ is obtained by considering sound-hard wall boundary condition $\mathbf{n} \cdot \nabla p=0$, which here implicates that at $r=a$, where $a$ is the radius of the duct wall,

$$
J_{m_{j}}^{\prime}\left(k_{r j} a\right)=0
$$

Axial wave number $k_{z j}$ is evaluated from the effective wave number $k$ and the radial wave number $k_{r j}$ by

$$
k_{z j}=\sqrt{k^{2}-k_{r j}^{2}}
$$




\section{B. Weak formulation with mode matching}

The solution of the Helmholtz equation Eq. (2) in the non-uniform part $\Omega_{B}$ is obtained by the finite element method. For this purpose, we present the weak formulation of the equation: Find $p_{B}=p_{r}+p_{i} i$, where $p_{r} \in H^{1}\left(\Omega_{B}\right), p_{i} \in H^{1}\left(\Omega_{B}\right)$, such that

$$
\int_{\Omega_{B}} \frac{1}{\rho}\left(\nabla p_{B} \cdot \nabla v-k^{2} p_{B} v\right) d V-\int_{\partial \Omega_{B}} \frac{1}{\rho} \mathbf{n} \cdot \nabla p_{B} v d S=0,
$$

for any $v \in H^{1}\left(\Omega_{B}\right)$; $\mathbf{n}$ is outward normal vector. Solutions $p_{A}$ and $p_{C}$ are coupled to $p_{B}$ by the boundary conditions

$$
\begin{array}{ll}
\mathbf{n} \cdot \nabla p_{B}=\mathbf{n} \cdot \nabla p_{A} & \text { on } \Gamma_{A}, \\
\mathbf{n} \cdot \nabla p_{B}=\mathbf{n} \cdot \nabla p_{C} & \text { on } \Gamma_{C}, \\
p_{B}=p_{A} & \text { on } \Gamma_{A}, \\
p_{B}=p_{C} & \text { on } \Gamma_{C} .
\end{array}
$$

These conditions impose the continuity of pressure and velocity over the interfaces $\Gamma_{A}$ and $\Gamma_{C}$.

The first two conditions Eqs. (12) and (13) and Neumann condition Eq. (3) can be incorporated in the weak form Eq. (11), leading to the equation

$$
\begin{aligned}
& \int_{\Omega_{B}} \frac{1}{\rho}\left(\nabla p_{B} \cdot \nabla v-k^{2} p_{B} v\right) d V-\int_{\Gamma_{A}} \frac{1}{\rho} \mathbf{n} \cdot \nabla p_{A} v d S \\
& -\int_{\Gamma_{C}} \frac{1}{\rho} \mathbf{n} \cdot \nabla p_{C} v d S=0 .
\end{aligned}
$$

In mode matching, the two other conditions Eqs. (14) and (15) are imposed in weak forms: Find $p_{A} \in Z_{A}, p_{B}$ and $p_{C} \in Z_{C}$ such that

$$
\begin{aligned}
& \int_{\Gamma_{A}}\left(p_{B}-p_{A}\right) \bar{\Phi}_{i} d S=0 \quad \text { and } \\
& \int_{\Gamma_{C}}\left(p_{B}-p_{A}\right) \bar{\Psi}_{i} d S=0
\end{aligned}
$$

for $\bar{\Phi}_{i} \in Z_{A}$ any and $\bar{\Psi}_{i} \in Z_{C}$, where test function spaces are defined as $Z_{A}=\operatorname{span}_{j=0, \ldots, \infty}\left\{\Phi_{j}(r, \quad \theta)\right\}$ and $Z_{C}$ $=\operatorname{span}_{j=0, \ldots, \infty}\left\{\Psi_{j}(r, \theta)\right\}$. In summary, the (continuous) hybrid formulation of the acoustic problem in the waveguide is given by Eqs. (16) and (17).

Mufflers often include perforated ducts and walls with very fine geometrical details. Direct treatment of such details with the finite element method is possible but usually leads to an unnecessarily fine mesh with high computational cost. A more practical approach is to approximate perforated walls with an interface boundary condition which imposes the continuity of normal velocity and discontinuity of pressure through the wall. The pressure jump $\Delta p$ through the wall is given by

$$
\Delta p=\rho c \xi u_{n},
$$

where $\xi$ is the non-dimensional transfer impedance of the wall and $u_{n}$ is the normal velocity. For the impedance $\xi$, we use the empirical relationship of Sullivan and Crocker ${ }^{21}$ given by

$$
\xi=\frac{0.006+i k(t+0.75 \delta)}{\phi},
$$

where $k$ is the wave number, $t$ is the wall thickness, $\delta$ is the hole diameter, and $\phi$ is the perforation porosity. The same condition is used, for example, in Mehdizadeh and Paraschivoiu. ${ }^{5}$ There are several different empirical formulas available for the impedance (e.g., see $\mathrm{Lee}^{22}$ ), but the one by Sullivan and Crocker ${ }^{21}$ is sufficient for our purposes.

Let us denote a perforated wall in the muffler by $\Sigma$ and assume that it separates the muffler into two distinct parts $\Omega_{B, 1}$ and $\Omega_{B, 2}$. The acoustic pressure $p_{B}$ becomes discontinuous over the interface $\Sigma$ and we denote the solutions in two parts by $p_{B, 1}$ and by $p_{B, 2}$. They both satisfy the Helmholtz equation separately and are coupled by the interface conditions on $\Sigma$. By the linearized Euler equation we know that the normal derivatives of the solutions $p_{B, 1}$ and $p_{B, 2}$ on $\Sigma$ are given by

$$
\mathbf{n}_{j} \cdot \nabla p_{B, j}=-i \rho \omega \mathbf{u} \cdot \mathbf{n}_{j} \quad j=1,2 .
$$

With this information and the boundary conditions on $\Sigma$ we can derive the weak formulation for $p_{B, 1}$ : Find $p_{B, 1}=p_{\mathrm{r}, 1}$ $+i p_{i, 1}$, where $p_{r, 1} \in H^{1}\left(\Omega_{B, 1}\right)$ and $p_{i, 1} \in H^{1}\left(\Omega_{B, 1}\right)$ such that

$$
\begin{gathered}
\int_{\Omega_{B, 1}} \frac{1}{\rho}\left(\nabla p_{B, 1} \cdot \nabla v-k^{2} p_{B, 1} v\right) d V-\int_{\Gamma_{A} \cap \partial \Omega_{B, 1}} \frac{1}{\rho} \mathbf{n} \cdot \nabla p_{A} v d S \\
-\int_{\Gamma_{C} \cap \partial \Omega_{B, 1}} \frac{1}{\rho} \mathbf{n} \cdot \nabla p_{C} v d S-i \frac{k}{\xi} \int_{\Sigma}\left(p_{B, 2}-p_{B, 1}\right) v d S=0
\end{gathered}
$$

for all $v \in H^{1}\left(\Omega_{B, 1}\right)$. A related weak formulation is obtained for $p_{B, 2}$.

\section{HYBRID NUMERICAL METHOD}

The hybrid numerical method developed by Kirby ${ }^{6}$ to simulate sound propagation in acoustic waveguides is based on the formulation of Eqs. (16) and (17). In the non-uniform region $\Omega_{B}$, the solution $p_{B}$ is approximated by the finite element method, while in the uniform regions the solutions are represented in terms of the modal basis functions. Finite element discretization proceeds by approximating the acoustic pressure in $\Omega_{B}$ by

$$
\begin{aligned}
p_{B}(x) & =\sum_{j=1}^{n} N_{j}(x) p_{j}=\left[N_{1}(x), \ldots, N_{n}(x)\right]\left[\begin{array}{c}
p_{1} \\
\vdots \\
p_{n}
\end{array}\right] \\
& =\mathbf{N}(x)^{T} \mathbf{p},
\end{aligned}
$$

where $N_{j}(x)$ are global trial functions for finite element mesh, $p_{j}$ are the nodal values of the acoustic pressure at node $j$ and $n$ is the number of nodes in $\Omega_{B}$. Galerkin method of weighted residuals proposes that $N_{j}(x)$ are used as test functions $v$. The approximation in Eq. (22) is next replaced in Eq. (16) to form equation 


$$
\begin{aligned}
& \int_{\Omega_{B}} \frac{1}{\rho}\left(\mathbf{\nabla} \mathbf{N} \cdot \mathbf{\nabla} \mathbf{N}^{T}-k^{2} \mathbf{N} \mathbf{N}^{T}\right) d V \mathbf{p} \\
& -\int_{\Gamma_{A}} \frac{1}{\rho} \mathbf{N} \mathbf{n} \cdot \nabla p_{A} d S-\int_{\Gamma_{C}} \frac{1}{\rho} \mathbf{N} \mathbf{n} \cdot \nabla p_{C} d S=0 .
\end{aligned}
$$

The modal representations of the solution $p_{A}$ and $p_{C}$ involve an infinite number of terms and for numerical computations only a finite number of modes can be included. For simplicity, we use the same number of modes in all terms of (4), and we denote it by $m$. The normal derivatives of $p_{A}$ and $p_{C}$ on the planes $\Gamma_{A}$ and $\Gamma_{C}$ are then of the form

$\mathbf{n} \cdot \nabla p_{A}(r, \theta, 0)=\frac{\partial}{\partial x} p_{A}(r, \theta, 0)=-i \sum_{j=0}^{m} \lambda_{j} A_{j} \Phi_{j}+i \sum_{j=0}^{m} \lambda_{j} F_{j} \Phi_{j}$,

$\mathbf{n} \cdot \nabla p_{C}(r, \theta, 0)=\frac{\partial}{\partial x^{\prime}} p_{C}(r, \theta, 0)=-i \sum_{j=0}^{m} \gamma_{j} B_{j} \Psi_{j}$

These derivatives are substituted in (23) to get

$$
\begin{aligned}
& \int_{\Omega_{B}} \frac{1}{\rho}\left(\mathbf{\nabla} \mathbf{N} \cdot \mathbf{\nabla} \mathbf{N}^{T}-k^{2} \mathbf{N} \mathbf{N}^{T}\right) d V \mathbf{p} \\
& -\int_{\Gamma_{A}} \frac{1}{\rho} \mathbf{N}\left(-i \sum_{j=0}^{m} \lambda_{j} A_{j} \Phi_{j}+i \sum_{j=0}^{m} \lambda_{j} F_{j} \Phi_{j}\right) d S \\
& -\int_{\Gamma_{A}} \frac{1}{\rho} \mathbf{N}\left(-i \sum_{j=0}^{m} \gamma_{j} B_{j} \Psi_{j}\right) d S=0 .
\end{aligned}
$$

If we introduce the matrices $\hat{\mathbf{H}}, \hat{\mathbf{K}}$, and $\mathbf{G}$ and vector $\tilde{\mathbf{f}}$ by

$$
\begin{aligned}
& \hat{H}_{i j}=i \lambda_{j} \int_{\Gamma_{A}} \frac{1}{\rho} N_{i} \Phi_{j} d S, \\
& \hat{K}_{i j}=i \gamma_{j} \int_{\Gamma_{C}} \frac{1}{\rho} N_{i} \Psi_{j} d S, \\
& G_{i j}=\int_{\Omega_{B}} \frac{1}{\rho}\left(\nabla N_{i} \cdot \nabla N_{j}-k^{2} N_{i} N_{j}\right) d V, \\
& \tilde{f}_{i}=i \sum_{j=0}^{m} \lambda_{j} \int_{\Gamma_{A}} \frac{1}{\rho} N_{i} \Phi_{j} d S F_{j},
\end{aligned}
$$

we can write $(25)$ in the matrix form

$$
\hat{\mathbf{H}} \mathbf{a}+\mathbf{G p}+\hat{\mathbf{K}} \mathbf{b}=\tilde{\mathbf{f}} .
$$

Here, vector a contains $m$ modal amplitudes $A_{i}$ and vector $\mathbf{b}$ contains $m$ modal amplitudes $B_{i}$. We remind that amplitudes $F_{i}$ correspond to the known incident acoustic wave and amplitudes $C_{i}$ are assigned to be 0 to impose the non-reflecting condition on $\Gamma_{C}$.

By replacing Eqs. (4) and (22) (with finite number $m$ of modes) into Eq. (17), we get

$$
\begin{aligned}
& \int_{\Gamma_{A}} \bar{\Phi}_{i} \mathbf{N}^{T} d S \mathbf{p}=\sum_{j=0}^{m} \int_{\Gamma_{A}} \Phi_{j} \bar{\Phi}_{i} d S A_{j}+\sum_{j=0}^{m} \int_{\Gamma_{A}} \Phi_{j} \bar{\Phi}_{i} d S F_{j} \\
& \int_{\Gamma_{A}} \bar{\Psi}_{i} \mathbf{N}^{T} d S \mathbf{p}=\sum_{j=0}^{m} \int_{\Gamma_{A}} \Psi_{j} \bar{\Psi}_{i} d S B_{j} .
\end{aligned}
$$

With the matrices $\mathbf{H}, \tilde{\mathbf{H}}, \mathbf{K}$, and $\tilde{\mathbf{K}}$ and vector $\mathbf{f}$ given by

$$
\begin{aligned}
H_{i j} & =\int_{\Gamma_{A}} \Phi_{j} \bar{\Phi}_{i} d S, \\
\tilde{H}_{i j} & =-\int_{\Gamma_{A}} \bar{\Phi}_{i} N_{j} d S, \\
K_{i j} & =\int_{\Gamma_{C}} \Psi_{j} \bar{\Psi}_{i} d S, \\
\tilde{K}_{i j} & =-\int_{\Gamma_{C}} \bar{\Psi}_{i} N_{j} d S, \\
f_{i} & =-\sum_{j=0}^{m} \int_{\Gamma_{A}} \Phi_{j} \bar{\Phi}_{i} d S F_{i} .
\end{aligned}
$$

Equation (28) can be written in the matrix form

$$
\begin{aligned}
& \mathbf{H a}+\tilde{\mathbf{H}} \mathbf{p}=\mathbf{f}, \\
& \mathbf{K b}+\tilde{\mathbf{K}} \mathbf{p}=\mathbf{0} .
\end{aligned}
$$

By definition, $\mathbf{H}$ and $\mathbf{K}$ are identity matrices and $f_{i}=-F_{i}$.

We can now combine Eqs. (27) and (30) to have a single block matrix equation

$$
\left[\begin{array}{ccc}
\mathbf{H} & \tilde{\mathbf{H}} & \mathbf{0} \\
\hat{\mathbf{H}} & \mathbf{G} & \hat{\mathbf{K}} \\
\mathbf{0} & \tilde{\mathbf{K}} & \mathbf{K}
\end{array}\right]\left[\begin{array}{l}
\mathbf{a} \\
\mathbf{p} \\
\mathbf{b}
\end{array}\right]=\left[\begin{array}{c}
\mathbf{f} \\
\tilde{\mathbf{f}} \\
\mathbf{0}
\end{array}\right] .
$$

This linear equation is solved by standard LU decomposition method to obtain the finite element solution $p_{B}$ and the modal amplitudes $\mathbf{a}$ and $\mathbf{b}$. With this information the transmission loss of the non-uniform component $\Omega_{B}$ is readily obtained by Eq. (7).

\section{MULTIOBJECTIVE SHAPE OPTIMIZATION OF MUFFLERS}

This article concentrates on optimal shape design of acoustic mufflers by combining a hybrid numerical simulation method with a genetic algorithm for multiobjective optimization. In this section, we describe the general procedure of model-based optimization which combines a simulation method with an optimization algorithm. We define the multiobjective shape optimization problem of an acoustic muffler and consider the implementational details of the optimization algorithm.

In model-based optimization, the aim is represented by a mathematical function called an objective function. For a specific aim, the corresponding function is usually defined in such a way that smaller values indicate better designs. The value of the objective function depends on the solution of the simulation model, which further depends on a certain number of design variables. These variables may be, for example, related to the shape of a device or its material parameters.

The main stages of a model-based optimization procedure in the case of a shape optimization problem are illustrated in Fig. 2 (left). A more detailed explanation of each stage is given in the following list.

1. Geometric parametrized model: The shape of the muffler needs to be described by a finite number of real-valued parameters. Optimization begins from some initial parameter values. 

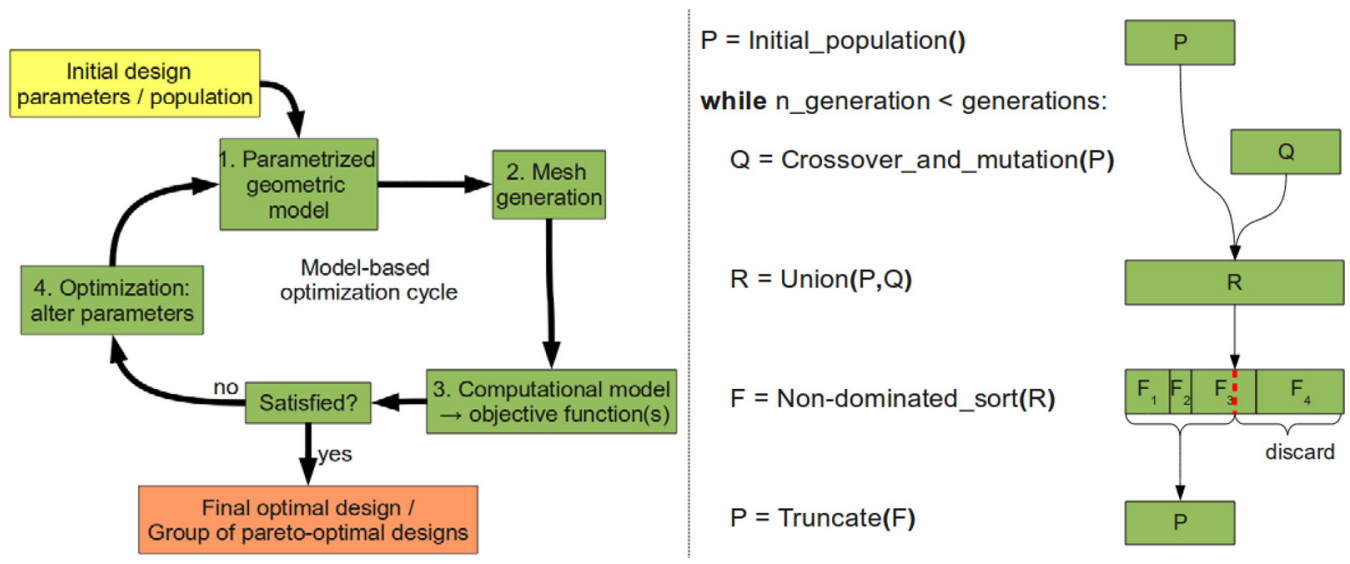

FIG. 2. (Color online) (left) Model-based optimization cycle and (right) simplified diagram of NSGA-II algorithm.

2. Mesh generation: Finite element mesh needs to be generated for the non-uniform (muffler) part of the geometry. The mesh is regenerated after each change in the geometry parameters.

3. Computational model: The hybrid numerical model is solved to obtain the transmission loss at certain frequency. Several different frequencies are computed to evaluate the objective functions.

4. Optimization: The optimization cycle is terminated if the current design is satisfactory. Otherwise an optimization algorithm is used to alter the shape parameters to improve the design with respect to the given objectives.

The model-based optimization procedure is modular in the sense that each stage can be realized with different methods or software. For example, it is possible to use different mesh generators in the second stage as long as the computational model is able to operate with the mesh format. On the other hand, the numerical model and the optimization algorithm can be chosen quite freely as long as there are suitable interfaces for the different components to communicate. The model-based optimization approach is flexible and by no means restricted to a specific simulation or optimization method.

In this work, we used the open-source Netgen mesh generator by $\mathrm{Schöberl}^{23}$ to create tetrahedral meshing of the muffler geometry. The hybrid numerical method considered in Sec. III was implemented using the Numerrin language, which is a modeling language for numerical computing by Numerola Ltd. As an optimization algorithm, NSGA-II of Deb et al $^{24}$ is considered, which is explained in Sec. IV B.

\section{A. Multiobjective optimization problem}

Often in industrial design, optimization should be performed with respect to multiple conflicting criteria. The general formulation of a multiobjective optimization problem reads as follows:

$$
\begin{gathered}
\min _{\mathbf{x} \in S} f_{1}(\mathbf{x}), \\
\vdots \\
\min _{\mathbf{x} \in S} f_{m}(\mathbf{x}),
\end{gathered}
$$

where $f_{i}: \mathbb{R}^{n} \rightarrow \mathbb{R}$ are objective functions that depend (often indirectly) on $\mathbf{x} \in S$, a vector consisting of design variables $x_{i}$ that belong to a feasible region $S$ of design space $\mathbb{R}^{n}$. For shape optimization problems, the vector $\mathbf{x}$ defines the shape of the geometry to be optimized. A solution is called Pareto optimal if no other solution exists that is better with respect to at least one objective function and equal with respect to other objective functions. Similarly, a solution is called nondominated, if no other solution within a group of (possibly non-optimal) solutions exists that is better with respect to at least one objective function and equal with respect to other objective functions.

The multiobjective optimization problem for acoustic mufflers is defined to maximize TL at two frequency ranges simultaneously. Both ranges correspond to a separate objective function $f$. Shape of the muffler is defined by the design parameters $\mathbf{x}$ and the frequency ranges are given by the vectors $\boldsymbol{\omega}$ and $\mathbf{\imath}$. Then, the objective functions are as follows:

$$
f_{1}(\mathbf{x})=-\frac{1}{n_{\omega}} \sum_{i=1}^{n_{\omega}} \tau\left(\mathbf{x}, \omega_{i}\right) \quad \text { and } \quad f_{2}(\mathbf{x})=-\frac{1}{n_{l}} \sum_{i=1}^{n_{l}} \tau\left(\mathbf{x}, l_{i}\right),
$$

where $\tau$ is given by

$$
\tau(\mathbf{x}, f)=\min \left(T L(\mathbf{x}, f), T L_{\max }\right) .
$$

Here, $T L(\mathbf{x}, f)$ is the TL value given by Eq. (7) for a design determined by $\mathbf{x}$ and $T L_{\max }$ is a limiting value for transmission loss, which is necessary due to possible narrow infinite peaks in transmission loss function that inhibit good convergence of the optimizer.

\section{B. NSGA-II genetic algorithm}

The non-dominated sorting genetic algorithm, NSGA-II introduced by Deb et al. ${ }^{24}$ is considered as a multi-objective optimization method. Genetic algorithms (GA) are stochastic optimization algorithms that mimic genetic drift and the Darwinian strife for survival. Unlike traditional gradient-based optimizers that need the derivatives and a good starting point, GAs have a good opportunity to locate the global 
optimum in a near optimal manner. In genetic algorithms, there is a population of individuals that correspond to unique design vectors. In our implementation, the initial population $P$ of $n_{\text {pop }}$ individuals is generated such that first, a random population of $10 n_{\text {pop }}$ individuals is generated, from which $n_{\text {pop }}$ best individuals are then chosen to the initial population. In the analogy of genetic evolution, crossover and mutation is used to evolve the population toward the optimum. In Fig. 2 (right), the basic structure of the algorithm is illustrated. In the following, the NSGA-II optimization algorithm implementation details are described briefly. The algorithm cycle consists of the following phases.

(1) Selection: Parents for crossover are selected by binary selection, i.e., two candidates are chosen at random and better candidate is selected to crossover.

(2) Crossover: Simulated binary crossover (SBX) operator of Agrawal and $\operatorname{Deb}^{25}$ is used to obtain children from chosen parents. SBX operator combines the properties $g_{1}, g_{2}$ from both parents according to the distribution

$$
\begin{aligned}
& c_{1}=\left[(1+\alpha) g_{1}+(1-\alpha) g_{2}\right] / 2, \\
& c_{2}=\left[(1-\alpha) g_{1}+(1+\alpha) g_{2}\right] / 2,
\end{aligned}
$$

where $\alpha$ is

$$
\alpha= \begin{cases}(2 u)^{1 /\left(\eta_{c}+1\right)} & \text { if } u \leq 0.5 \\ 1 /[2(1-u)]^{1 /\left(\eta_{c}+1\right)} & \text { if } u>0.5\end{cases}
$$

for random variable $u \in[0,1]$. Crossover distribution index $\eta_{c}$ determines how big the difference between children and parents is likely to become, the bigger $\eta_{c}$ makes the operator produce more unique children. The crossover operator is applied to parents with probability $p_{c}$. Values $p_{c}=0.9$ and $n_{c}=20$ are used in all optimization problems of Sec. V.

(3) Mutation: Children genes are mutated with a polynomial mutation operator of Agrawal and Deb. ${ }^{25}$ The mutation alters the design variables according to formula $g_{\text {new }}=g_{\text {old }}+\delta(r)\left(f_{\max }-f_{\text {min }}\right)$, where $\delta(r)$ chosen according to distribution

$$
\delta(r)= \begin{cases}(2 r)^{1 /\left(\eta_{m}+1\right)}-1 & \text { if } r<\frac{1}{2} \\ 1-(2 r)^{1 /\left(\eta_{m}+1\right)}-1 & \text { if } r \geq \frac{1}{2}\end{cases}
$$

where $r \in[0,1]$ is a random number and $\eta_{m}$ is the mutation distribution index determining the impact of mutation operator. Mutation operator is applied with probability $p_{m}$. Values $p_{m}=0.25$ and $\eta_{m}=10$ are used in all optimization problems of Sec. V.

The child population $Q$ [see Fig. 2 (right)] is obtained by steps $1-3$ and the objective functions are evaluated. Then populations $Q$ and $R$ are mixed to form a population $R$ which is then sorted using the non-dominated sorting algorithm of Deb et al. ${ }^{24}$ to rank the individuals according to their mutual domination. Then, the best half of $R$ is used as a new population $P$ in the next generation.

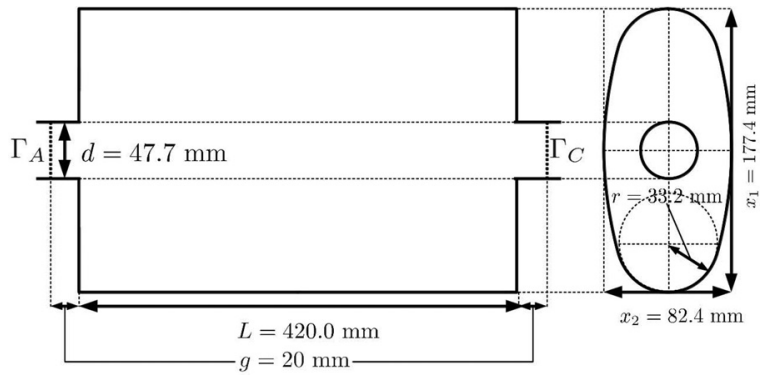

FIG. 3. The diagram of the muffler component used to validate and evaluate the hybrid numerical model.

\section{NUMERICAL EXPERIMENTS}

\section{A. Validation of the hybrid model}

The hybrid numerical method is validated by comparing the numerically evaluated transmission loss values to the experimental results reported by Gerges et al. ${ }^{8}$ The first test in Gerges et $a l^{8}$ is a simple expansion chamber which is illustrated and defined in Fig. 3. The transmission loss values of this geometry are evaluated with the hybrid numerical method for the same frequency range as in Gerges et al. ${ }^{8}$ The numerical and experimental results are plotted in Fig. 4. There is a clear consistency between the results, and it can be concluded that the hybrid method predicts the transmission loss behavior very accurately in chosen test problem. Gerges et $\mathrm{al}^{8}{ }^{8}$ reports also TL values obtained with the transfer matrix method, which gives accurate results for frequencies below $2000 \mathrm{~Hz}$. However, above $2000 \mathrm{~Hz}$ the results of the transfer matrix method differ significantly from the experimental results, whereas the hybrid method gives reasonable accuracy over the whole frequency range.

Table I reports TL values of the expansion chamber computed by the hybrid method for two frequencies. The number $m$ of modes in the modal representations $p_{A}$ and $p_{C}$ is gradually increased to study how many are needed for sufficient accuracy. With both frequencies, there is not a big change in these values as the number of modes $m$ increases. As the frequency is low such that there is only one propagating mode, it is not necessary to use more than one mode to

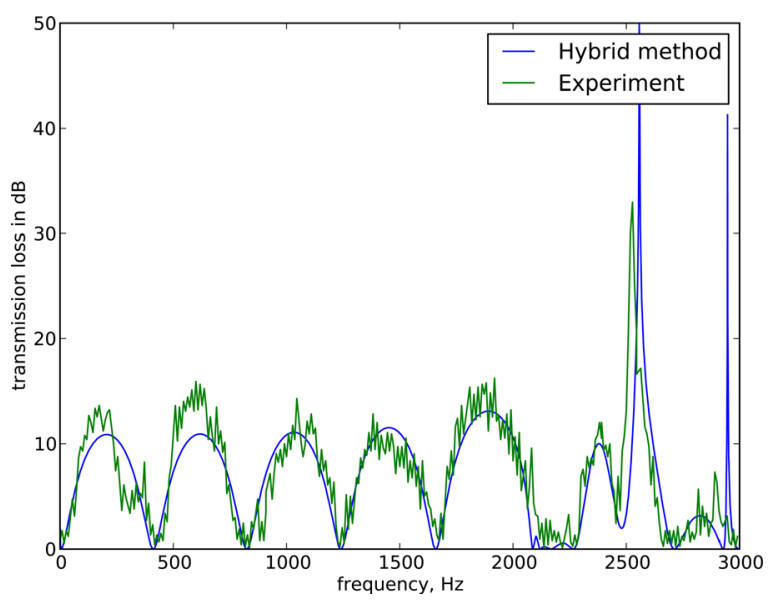

FIG. 4. (Color online) Numerically evaluated transmission loss compared to experimental results with the expansion chamber of Fig. 3. 
TABLE I. The transmission loss of expansion chamber with respect to number $m$ of modes.

\begin{tabular}{lcc}
\hline \hline$m$ & $500 \mathrm{~Hz}$ & $2900 \mathrm{~Hz}$ \\
\hline 1 & 7.346236753 & 1.281625853 \\
2 & 7.346236753 & 1.281639638 \\
3 & 7.346236794 & 1.281639526 \\
4 & 7.349007703 & 1.281832511 \\
5 & 7.349007776 & 1.281832527 \\
6 & 7.349008748 & 1.281832764 \\
7 & 7.349008866 & 1.281832732 \\
8 & 7.349009086 & 1.281832982 \\
9 & 7.349009174 & 1.281833040 \\
10 & 7.354649180 & 1.282237512 \\
\hline
\end{tabular}

get reasonably accurate results. The accuracy is limited also by the spatial discretization mesh, so we can not expect full convergence to a specific value just by increasing $m$. In order to improve accuracy of the transmission loss evaluation, it would be necessary also to use finer mesh and/or higher order integration formula on the inlet/outlet interfaces. Otherwise, there is no advantage of using additional modes. In optimization test cases, 15 wave modes, $m=15$, are considered.

\section{B. Optimization problem \#1}

The dimensions and a schematic illustration of the first optimization case representing a muffler with a perforated duct are given in Fig. 5. Geometry of the muffler is determined by eight parameters, four of which are fixed, and four are varied to optimize the transmission loss. The fixed parameters are as follows.

(1) Second axis of the elliptic cross section, $H=177.4 \mathrm{~mm}$.

(2) Diameter of the perforated duct, $d=47.7 \mathrm{~mm}$.

(3) Thickness of the perforated duct, $t=1.55 \mathrm{~mm}$.

(4) The distance determining the locations of truncation boundaries $\Gamma_{A}$ and $\Gamma_{C} \mathrm{~mm}, g=20 \mathrm{~mm}$.

The four design variables $x_{i}$ with their admissible ranges are as follows.

(1) Length of the chamber, $x_{1} \in[300,500] \mathrm{mm}$.

(2) First axis of the elliptic cross section, $x_{2} \in[82.4,177.4]$ $\mathrm{mm}$.

(3) Porosity of the perforated duct, $x_{3}=\phi \in[0.01,0.1]$.

(4) Pore diameter of the perforated duct, $x_{4}=\delta \in[1,5] \mathrm{mm}$.

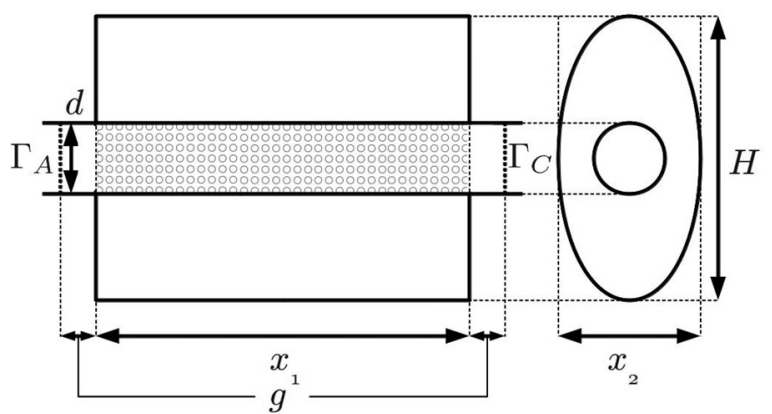

FIG. 5. The diagram of a muffler component used in optimization problem \#1.

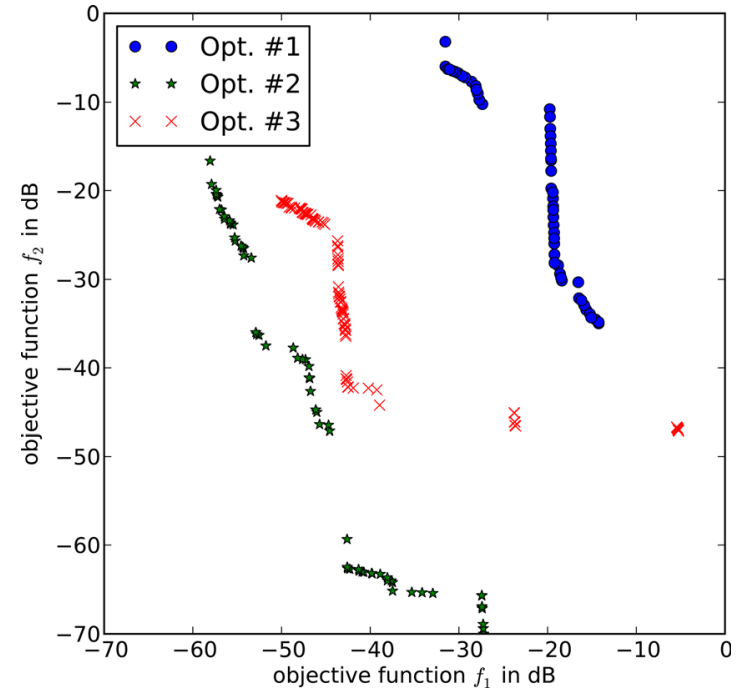

FIG. 6. (Color online) The non-dominated fronts for optimization test cases $\# 1, \# 2$ and \#3.

The following material parameters are used in all test cases.

(1) The speed of sound, $c=343.3 \mathrm{~m} / \mathrm{s}$.

(2) Density of air at temperature $20^{\circ} \mathrm{C}, \rho=1.2 \mathrm{~kg} / \mathrm{m}^{3}$.

Transmission loss is maximized for the frequency ranges $1200-1300 \mathrm{~Hz}$ and $1900-2000 \mathrm{~Hz}$ by choosing $\boldsymbol{\omega}=[1200,1250,1300] \mathrm{Hz}$ and $\mathbf{\imath}=[1900,1925,1950,1975$, 2000] $\mathrm{Hz}$ in the formulation Eq. (33). The limiting value in objective function (34) is given by $T L_{\max }=50 \mathrm{~dB}$. The population size $n_{\text {pop }}=50$ was used and the average values of the objective functions in the initial population were $f_{1}=-11.7$ $\mathrm{dB}$ and $f_{2}=-0.6 \mathrm{~dB}$.

Figure 6 presents the non-dominated fronts (approximation of Pareto optimal fronts) of all optimization test problems (\#1, \#2 and \#3) that are obtained by NSGA-II algorithm after 100 generations. In all test cases, optimization converged mainly to the same front with different tested random number generator seed numbers, which implicates that the algorithm is behaving robustly.

It can be seen that after optimization, the objectives are clearly better than on average before optimization. In Fig. 7, the transmission loss as a function of frequency is plotted for an optimal solution that is chosen from the non-dominated front at point $f_{1}=-18.4 \mathrm{~dB}, f_{2}=-30.1 \mathrm{~dB}$ which corresponds to the design vector $\mathbf{x}=[492 \mathrm{~mm}, 87.6 \mathrm{~mm}, 0.097$, $1.88 \mathrm{~mm}]$.

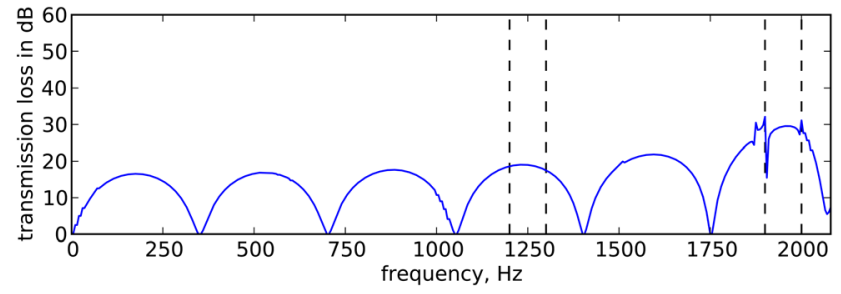

FIG. 7. (Color online) The transmission loss versus frequency for the optimization case \#1 with $f_{1}=-18.4 \mathrm{~dB}, f_{2}=-30.1 \mathrm{~dB}$ is plotted. It corresponds to the design variable values $x_{1}=492 \mathrm{~mm}, x_{2}=87.6 \mathrm{~mm}$, $x_{3}=\phi=0.097$, and $x_{4}=\delta=1.88 \mathrm{~mm}$. 


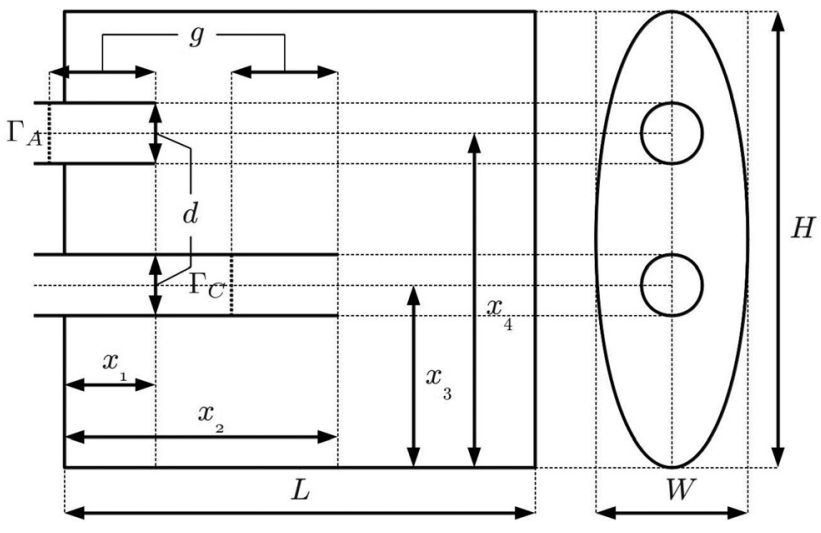

FIG. 8. The diagram of a muffler component used in optimization test case \#2.

\section{Optimization problem \#2}

The second optimization test case represents a reverse flow chamber. The dimensions and a schematic illustration of the test case are given in Fig. 8. Geometry of the muffler is determined by 10 parameters, six of which are fixed, and four are varied to optimize the transmission loss. The fixed parameters are as follows.

(1) The length of the chamber, $L=200 \mathrm{~mm}$.

(2) The inner diameter of the ducts, $d=47.7 \mathrm{~mm}$.

(3) Thickness of ducts is $1.55 \mathrm{~mm}$.

(4) The first axis of the cylindrical chamber, $W=60 \mathrm{~mm}$.

(5) The second axis of the cylindrical chamber, $H=100 \mathrm{~mm}$.

(6) The distance determining the locations of truncation boundaries $\Gamma_{A}$ and $\Gamma_{C} \mathrm{~mm}, g=20 \mathrm{~mm}$.

Four shape parameters with their admissible ranges are as follows.

(1) The lengths of inlet duct inside the chamber, $x_{1}$ $\in[80,130] \mathrm{mm}$.

(2) The length of outlet duct inside the chamber $x_{2}$ $\in[80,130] \mathrm{mm}$.

(3) The location of inlet duct $x_{3} \in[-70,-27.5] \mathrm{mm}$.

(4) The location of outlet duct $x_{4} \in[27.5,70] \mathrm{mm}$.

Transmission loss is maximized for the frequency ranges $1550-1600 \mathrm{~Hz}$ and $2450-2500 \mathrm{~Hz}$ by choosing $\boldsymbol{\omega}=[1550,1560,1570,1580,1590,1600] \mathrm{Hz}$ and $\mathbf{\imath}=[2450$, $2460,2470,2480,2490,2500] \mathrm{Hz}$ in the formulation Eq.

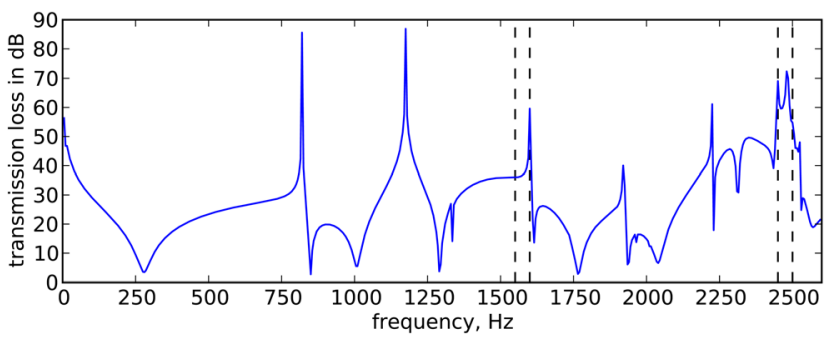

FIG. 9. (Color online) The transmission loss versus frequency for the optimization case \#2 with $f_{1}=-42.5 \mathrm{~dB}, f_{2}=-62.7 \mathrm{~dB}$ is plotted. It corresponds to the design variable values $x_{1}=94.7 \mathrm{~mm}, x_{2}=126 \mathrm{~mm}$, $x_{3}=-56.3 \mathrm{~mm}$ and $x_{4}=27.5 \mathrm{~mm}$.

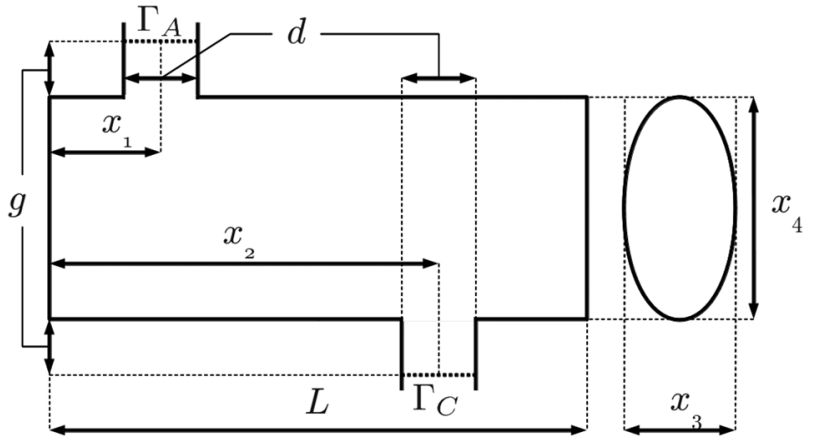

FIG. 10. The diagram of a muffler component used in optimization problem \#3.

(33). The limiting value in objective function (34) is given by $T L_{\max }=70 \mathrm{~dB}$. The population size $n_{\mathrm{pop}}=100$ was used and the average values of the objective functions in the initial population were $f_{1}=-21.3 \mathrm{~dB}$ and $f_{2}=-19.67 \mathrm{~dB}$.

It can be seen that for optimization case \#2, the objective function values are over $20 \mathrm{~dB}$ better than with the initial parameter values. In Fig. 9, the transmission loss as a function of frequency is plotted for an optimal solution that is chosen from the non-dominated front at point $f_{1}=-42.5 \mathrm{~dB}$, $f_{2}=-62.7 \mathrm{~dB}$ which corresponds to the design vector $\mathbf{x}=[94.7,126,-56.3,27.5] \mathrm{mm}$.

\section{Optimization problem $\# 3$}

The dimensions and a schematic illustration of the third optimization problem muffler component are given in Fig. 10. Geometry of the muffler is determined by seven parameters, three of which are fixed, and four are varied to optimize the transmission loss. The fixed parameters are as follows.

(1) The length of the chamber, $L=500 \mathrm{~mm}$.

(2) The diameter of inlet and outlet ducts, $d=67.2 \mathrm{~mm}$.

(3) The distance determining the locations of truncation boundaries $\Gamma_{A}$ and $\Gamma_{C} \mathrm{~mm}, g=100 \mathrm{~mm}$.

The four design variables $x_{i}$ with their admissible ranges are as follows.

(1) The location of inlet duct, $x_{1} \in[d / 2, L-d / 2]=[33.6$, 466.4] $\mathrm{mm}$.

(2) The location of outlet duct, $x_{2} \in[L / 2, L-d / 2]=[250$, $466.4] \mathrm{mm}$.

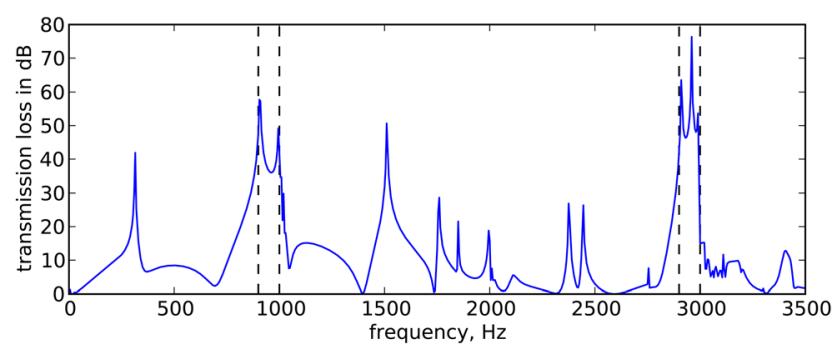

FIG. 11. (Color online) The transmission loss versus frequency for the optimization case \#3 with $f_{1}=-42.5 \mathrm{~dB}, f_{2}=-42.2 \mathrm{~dB}$ is plotted. It corresponds to the design variable values $x_{1}=410 \mathrm{~mm}, x_{2}=275 \mathrm{~mm}, x_{3}=110$ and $x_{4}=110 \mathrm{~mm}$. 


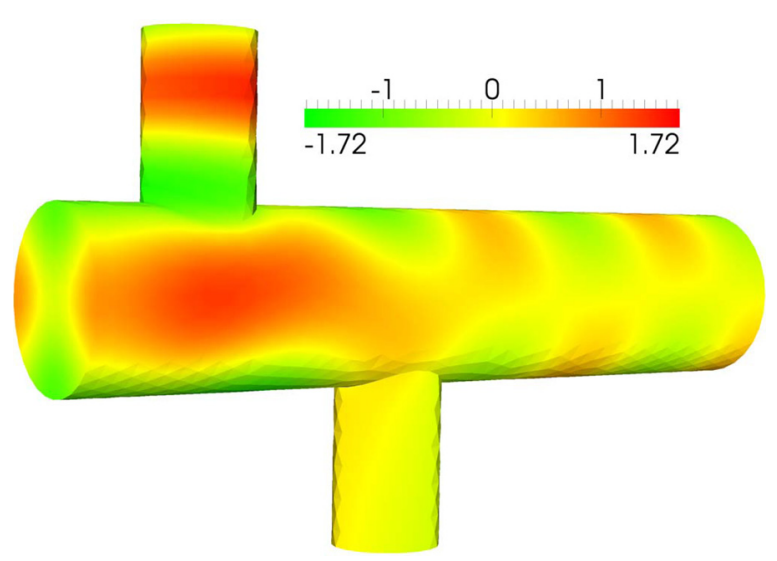

FIG. 12. (Color online) The real part of sound pressure for optimal solution of problem \#3 at $f=2950 \mathrm{~Hz}$.

(3) The length of the first axis of chamber's elliptic cross section, $x_{3} \in[90,120] \mathrm{mm}$.

(4) The length of the second axis of chamber's elliptic cross section, $x_{4} \in[90,120] \mathrm{mm}$.

Transmission loss is maximized in frequency ranges 900-1000 Hz and $2900-3000 \mathrm{~Hz}$ by choosing $\boldsymbol{\omega}=[900,925$, $950,975,1000] \mathrm{Hz}$ and $\mathbf{l}=[2900,2925,2950,2975,3000]$ $\mathrm{Hz}$ in the formulation Eq. (33). The limiting value in objective function (34) is given by $T L_{\max }=50 \mathrm{~dB}$. The population size $n_{\text {pop }}=200$ was used and the average values of the objective functions in the initial population were $f_{1}=-14.1 \mathrm{~dB}$ and $f_{2}=-9.1 \mathrm{~dB}$.

It can be seen that for optimization case \#3, the objective function values are over $20 \mathrm{~dB}$ better than with the initial parameter values. In Fig. 11, the transmission loss as a function of frequency is plotted for an optimal solution that is chosen from the non-dominated front at point $f_{1}=-42.5 \mathrm{~dB}$, $f_{2}=-42.2 \mathrm{~dB}$ which corresponds to the design vector $\mathbf{x}=[410,275,110,110] \mathrm{mm}$. The transmission loss is very good at both optimized frequency ranges. The same parameters are used also to plot the example solution in Fig. 12, which shows the real part of the sound pressure.

\section{CONCLUSIONS}

Multiobjective shape optimization of acoustic mufflers was considered. The shape parameters of the muffler were varied to maximize the sound transmission loss. NSGA-II was used as an multiobjective optimization method, and acoustic attenuation was computed by a hybrid numerical method, which combines finite element method in the nonuniform muffler geometry to a modal representation of the solution in the uniform inlet and outlet ducts.

Two frequency ranges were optimized simultaneously. For each test problem, example solutions were chosen from the non-dominated front that was given by NSGA-II optimizer algorithm. The transmission loss as a function of frequency was plotted for them to evaluate the optimization results. Desired attenuation properties were reached in all test cases. The optimization significantly improved the objective functions when compared to the initial random designs.
The hybrid method provides accurate and realistic modeling of acoustics in muffler component. Finite element method allows almost arbitrary three-dimensional shape of the design and boundary conditions, which brings versatile possibilities to the formulation of muffler component optimization. Combined with the NSGA-II optimization algorithm, the method offers a generic, robust, and advanced approach to many three-dimensional muffler optimization problems.

\section{ACKNOWLEDGMENTS}

Authors want to thank Dr. Jari Toivanen and Dr. Janne Martikainen for valuable cooperation during the manuscript preparation and Numerola Oy for providing the Numerrin modeling software.

${ }^{1}$ H. Bodén and R. Glav, Handbook of Noise and Vibration Control (John Wiley and Sons, Hoboken, NJ, 2007), Chap. 85.

${ }^{2}$ J. W. Lee and Y. Y. Kim, “Topology optimization of muffler internal partitions for improving acoustical attenuation performance," Int. J. Numer. Methods Eng. 80, 455-477 (2009).

${ }^{3}$ M. L. Munjal, Acoustics of Ducts and Mufflers with Application to Exhaust and Ventilation System, Design (John Wiley and Sons, Hoboken, NJ, 1987), Chap. 2.

${ }^{4} \mathrm{~S}$. Bilawchuk and K. R. Fyfe, "Comparison and implementation of the various numerical methods used for calculating transmission loss in silencer systems," Appl. Acoust. 64, 903-916 (2003).

${ }^{5} \mathrm{O}$. Z. Mehdizadeh and M. Paraschivoiu, "A three-dimensional finite element approach for predicting the transmission loss in mufflers and silencers with no mean flow," Appl. Acoust. 66, 902-918 (2005).

${ }^{6} \mathrm{R}$. Kirby, "Modeling sound propagation in acoustic waveguides using a hybrid numerical method,” J. Acoust. Soc. Am. 124, 1930-1940 (2008).

${ }^{7}$ R. J. Astley, "FE mode-matching schemes for the exterior Helmholtz problem and their relationship to the FE-DtN approach," Commun. Numer. Methods Eng. 12, 257-267 (1996).

${ }^{8}$ S. N. Y. Gerges, R. Jordan, F. A. Thieme, J. L. B. Coelho, and J. P. Arenas, "Muffler modeling by transfer matrix method and experimental verification,” J. Braz. Soc. Mech. Sci. Eng. 27, 132-140 (2005).

${ }^{9}$ R. Bernhard, "Shape optimization of reactive mufflers," Noise Control Eng. 27, 10-18 (1986).

${ }^{10}$ R. Barbieri and N. Barbieri, "Finite element acoustic simulation based shape optimization of a muffler," Appl. Acoust. 67, 346-357 (2006).

${ }^{11}$ C. Kuskinen, A. Riveros, and S. Floody, "Shape optimization of reactivedissipative mufflers,” J. Acoust. Soc. Am. 128, 2367-2367 (2010).

${ }^{12}$ M.-C. Chin, L.-J. Yeh, Y.-C. Chang, and L. Tian-Syung, "Shape optimization of single-chamber mufflers with side inlet/outlet by using boundary element method, mathematic gradient method and genetic algorithm," Tamkang J. Sci. Eng. 12, 85-98 (2009).

${ }^{13}$ L.-J. Yeh, Y.-C. Chang, and M.-C. Chiu, "Shape optimal design on double-chamber mufflers using simulated annealing and a genetic algorithm," Turkish J. Eng. Env. Sci. 29, 207-224 (2005).

${ }^{14}$ Y.-C. Chang, M.-C. Chiu, and L.-W. Wu, "Shape optimization of mufflers hybridized with multiple connected tubes using the boundary element method, neural networks, and genetic algorithm," Proc. Inst. Mech. Eng. Part C 224, 901-913 (2010).

${ }^{15}$ Y.-C. Chang and M.-C. Chiu, "Shape optimization of one-chamber perforated plug/non-plug mufflers by simulated annealing method," Int. J. Numer. Methods Eng. 74, 1592-1620 (2008).

${ }^{16}$ Y.-C. Chang and M.-C. Chiu, "Optimization of multi-chamber mufflers with reverse-flow ducts by algorithm of simulated annealing," Arch. Acoust. 35, 13-33 (2010).

${ }^{17}$ M.-C. Chiu, "Shape optimization of one-chamber mufflers with reverseflow ducts using a genetic algorithm," J. Mar. Sci. Technol. 18, 12-23 (2010).

${ }^{18}$ S. W. Rienstra and A. Hirschberg, An Introduction to Acoustics (Eindhoven University of Technology, Eindhoven, Netherlands, 2010), Chap. 2.

${ }^{19}$ R. Kirby, "A comparison between analytic and numerical methods for modelling automotive dissipative silencers with mean flow," J. Sound Vib. 325, 565-582 (2009). 
${ }^{20}$ R. Kirby and J. Lawrie, "A point collocation approach to modelling large dissipative silencers," J. Sound Vib. 286, 313-339 (2005).

${ }^{21}$ J. W. Sullivan and M. J. Crocker, "Analysis of concentric-tube resonators having unpartitioned cavities," J. Acoust. Soc. Am. 64, 207-215 (1978).

${ }^{22}$ I. Lee, "Acoustic characteristics of perforated dissipative and hybrid silencers," Ph.D. thesis, OH State University, Columbus, OH, 2005.
${ }^{23}$ J. Schöberl, "NETGEN - An advancing front 2D/3D-mesh generator based on abstract rules," Comput. Visual. Sci. 1, 41-52 (1997).

${ }^{24}$ K. Deb, A. Pratap, S. Agarwal, and T. Meyarivan, "A fast elitist multiobjective genetic algorithm: NSGA-II,” IEEE T. Evolut. Comput. 6, 182197 (2000).

${ }^{25}$ R. B. Agrawal and K. Deb, "Simulated binary crossover for continuous search space," Complex Syst. 9, 115-148 (1995). 